\title{
Bilateral carotid body paragangliomas
}

\author{
Shih-Hao Wang MD, Kuan-Ming Chiu MD PhD, Po-Wen Cheng MD PhD
}

Competing interests: None

This article has been peer reviewed.

Affiliations: From the Department of Otolaryngology (Wang), National Taiwan University Hospital; and the

Cardiovascular Center (Chiu) and the Department of Otolaryngology (Cheng), Far Eastern Memorial Hospital, Taipei, Taiwan

Correspondence to: Dr. Po-Wen Cheng, powenjapan@yahoo.com.tw

CMAJ 2011. DOI:10.1503 /cmaj.092114 declared.

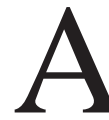
42-year-old man presented with bilateral, nontender, nonmobile masses in his neck, which had been present for several months. Magnetic resonance imaging (MRI) (Figure 1A) and magnetic resonance angiography (MRA) (Figure 1B) showed bilateral, intensely enhancing lesions that caused splaying of external and internal carotid arteries. This finding was consistent with a diagnosis of bilateral carotid body paragangliomas. After transarterial embolization, the tumours were surgically excised, following ligation of the respective external carotid artery. The preoperative diagnosis was confirmed histopathologically (Appendix 1, available at www.cmaj.ca/cgi/content/full/cmaj.092114/DC1). The possibility of a familial syndrome was excluded by family history.

Carotid body paragangliomas often manifest as slow-growing, nontender masses in the neck, located anterior to the sternocleidomastoid muscle at the level of the hyoid bone. They are movable in the horizontal plane, but their mobility is limited in the vertical plane (Fontaine sign). Occasionally, a carotid pulse, bruit or thrill may be associated with the mass. Symptoms such as fluc-
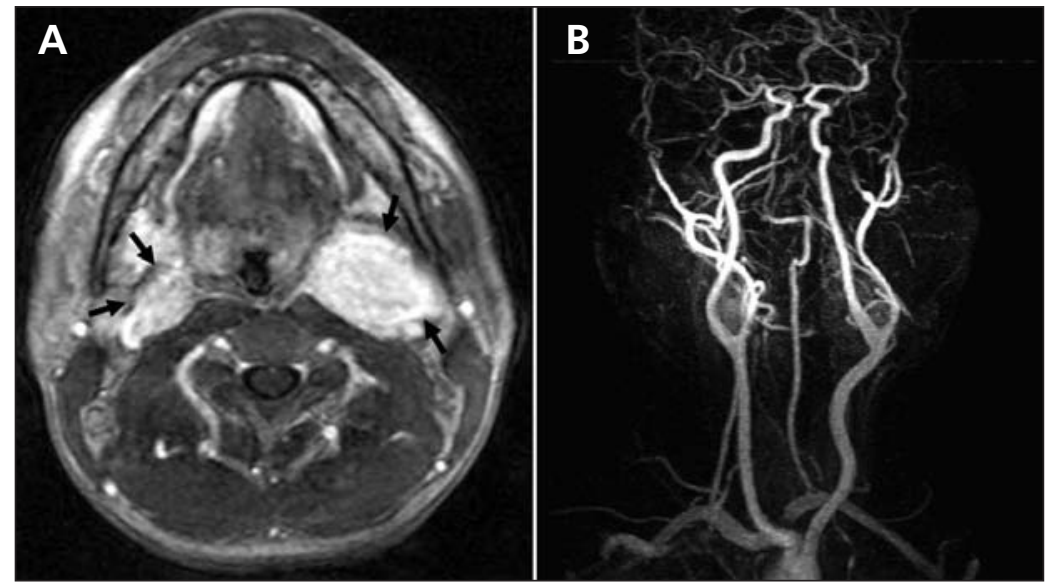

Figure 1: (A) Axial $T_{1}$-weighted magnetic resonance (MR) image, with gadolinium enhancement, of the neck of a 42-year-old man showing bilateral, well-circumscribed masses (arrows) with a "salt and pepper" appearance; masses measured $2.0 \times 1.6 \mathrm{~cm}$ (right) and $4.0 \times 3.3 \mathrm{~cm}$ (left). (B) MR angiogram showing splaying of external and internal carotid arteries (lyre sign), bilaterally. tuating hypertension, blushing and palpitations suggest the tumour may be producing catecholamines. Bilateral involvement is uncommon but occurs more frequently in familial cases.

Prebiopsy imaging of a neck mass in the submandibular area is essential: a biopsy of a carotid body paraganglioma may cause uncontrollable hemorrhage. The characteristic MRI shows lowsignal intensity in $T_{1}$-weighted sequences and a hyperintense signal in $T_{2}$-weighted sequences. ${ }^{1}$ In our patient, the "salt and pepper" appearance represented a signal void, because of high blood flow within the mass. Contrast-enhanced MRA combined with MRI is more accurate than MRI alone. ${ }^{2}$ The pathognomonic angiographic finding is widening of the carotid bifurcation by a welldefined tumour blush (lyre sign).

Although preoperative embolization may reduce tumour vascularity and decrease intraoperative blood loss, it increases the risk of cerebral ischemia and cranial nerve palsy. ${ }^{2}$ Complete surgical excision is the treatment of choice because the incidence rate for malignant degeneration of these tumours may be as high as $10 \% .^{1}$ This degeneration cannot be predicted by the initial clinical presentation or histological appearance. Although our patient underwent simultaneous surgical resection for both paragangliomas uneventfully, the recommended procedure is staged, not simultaneous, excision ${ }^{3}$ because surgical excision carries a high risk of neurologic damage, cerebrovascular complications and mortality. ${ }^{4}$

\section{References}

1. Wieneke JA, Smith A. Paraganglioma: carotid body tumor. Head Neck Pathol 2009;3:303-6.

2. Rao AB, Koeller KK, Adair CF. From the archives of the AFIP Paragangliomas of the head and neck: radiologic-pathologic correlation. Armed Forces Institute of Pathology. Radiographics 1999; 19:1605-32.

3. Kaman L, Singh R, Aggarwal R, et al. Diagnostic and therapeutic approaches to carotid body tumours: report of three cases and review of the literature. Aust N Z J Surg 1999;69:852-5.

4. Lim J-Y, Kim J, Kim SH, et al. Surgical treatment of carotid body paragangliomas: outcomes and complications according to the Shamblin classification. Clin Exp Otorhinolaryngol 2010;3: 91-5. 